\title{
ON THE $q$-ANALOGUE OF A BLACK BODY RADIATION
}

\author{
P. BABINEC \\ Department of Biophysics and Chemical Physics, Comenius University, MFF \\ Mlynska Dolina, 84215 Bratislava, Czecho-Slovakia
}

(Received July 6, 1992)

A statistical distribution function and spectral energy density are derived for the $q$-analogue of a black body radiation. These functions are different from the usual ones for $q \neq 1$ and they have singularities. The reason for these singularities is also discussed.

PACS numbers: $42.50 . \mathrm{Md}, 03.65 . \mathrm{Fd}, 11.30 . \mathrm{Na}$

Quantum algebras [1,2] have recently attracted a lot of attention in physics. In particular, the realization of the quantum algebra $\mathrm{SU}_{q}(2)$ in terms of the $q$-analoque of the quantum harmonic oscillator $[3,4]$ has initiated much work on this topic $[5,6]$.

A $q$-deformation of the Heisenberg algebra generated by a $q$-creation operator $a_{q}^{+}, q$-annihilation operator $a_{q}$, identity operator and a $q$-number operator $N_{q}$ in the $q$-analoque of the Hilbert space $\mathcal{H}_{q}$ with the $q$-boson vacuum $|0\rangle_{q}$ defined as $a_{q}|0\rangle_{q}=0$. The algebraic relations for the operators $a_{q}, a_{q}^{+}$and $N_{q}$ are

$$
\begin{aligned}
& a_{q} a_{q}^{+}-q a_{q}^{+} a_{q}=q^{-N q}, \\
& {\left[N_{q}, a_{q}\right]=-a_{q}, \quad\left[N_{q}, a_{q}^{+}\right]=a_{q}^{+} .}
\end{aligned}
$$

The operators $a_{q}, a_{q}^{+}$and $N_{q}$ act in $\mathcal{H}_{q}$ with the basis $|n\rangle_{q}(n=0,1,2, \ldots)$

$$
\begin{aligned}
& a_{q}|0\rangle_{q}=0, \\
& |n\rangle_{q}=\frac{\left(a^{+}\right)^{n}}{\left([n]_{q} !\right)^{1 / 2}}|0\rangle_{q},
\end{aligned}
$$

where the $q$-number is defined as

$$
[x]_{q}=\frac{q^{x}-q^{-x}}{q-q^{-1}}
$$

and the $q$-factorial

$$
[n]_{q} !=[n]_{q}[n-1]_{q} \ldots[1]_{q} .
$$


From the above it follows that

$$
\begin{aligned}
& a_{q}^{+}|n\rangle_{q}=[n+1]_{q}^{1 / 2}|n+1\rangle_{q}, \\
& a_{q}|n\rangle_{q}=[n]_{q}^{1 / 2}|n-1\rangle_{q} .
\end{aligned}
$$

The $q$-oscillator Hamiltonian can be introduced as

$$
H_{q}=\frac{P_{q}^{2}}{2 m}+\frac{m \omega^{2}}{2} X_{q}^{2},
$$

which can also be written in terms of the creation and annihilation operators

$$
H_{q}=\frac{1}{2} \hbar \omega\left(a_{q}^{+} a_{q}+a_{q} a_{q}^{+}\right) .
$$

Its eigenvalues are then

$$
E_{N}=\frac{1}{2} \hbar \omega\left([N]_{q}+[N+1]_{q}\right), \quad N=0,1,2 \ldots
$$

Recently the statistical distribution function has been calculated for the $q$-deformed harmonic oscillator for a given eigenvalue $N$ of $N_{q}$ using the double time Green function technique [7].

The result is

$$
\bar{n}(q, N)=\frac{q^{-N}}{\exp [\beta \hbar \omega A(q)]-q},
$$

where $A(q)=\frac{1}{2}(1+q) \cosh [(2 N+1) \ln q] / \cosh \left(\frac{1}{2} \ln q\right)$.

The spectral energy density is given by

$$
U(\omega, q, N)=\bar{n}(q, N) \hbar \omega \frac{\omega^{2}}{\pi^{2} c^{3}} .
$$

We can as usualy, introduce a dimensionless variable $x=\beta \hbar \omega$ and a dimensionless function $\bar{U}(x, q, N)=\pi^{2} \hbar^{2}(c \beta)^{3} U(\omega, q, N)$

$$
\bar{U}(x, q, N)=\frac{x^{3} q^{-N}}{\exp [x A(q)]-q} .
$$

This function has a singularity for

$$
x_{\mathrm{c}}=\frac{\ln q}{A(q)} \text {. }
$$

The aim of this paper is to calculate the distribution function and spectral energy density for a $q$-boson field (black body radiation) without restriction to the fixed eigenvalue of $N_{q}$. Approximately this function has been studied in Ref. [8].

As pointed out in Ref. [3] spontaneous and stimulated emission for $q$-boson fields originates in the $q$-boson factors

$$
a_{q}^{+} \rightarrow\left([n+1]_{q}\right)^{1 / 2}
$$

for creation and

$$
a_{q} \rightarrow\left([n]_{q}\right)^{1 / 2}
$$

for stimulated absorption.

Let us now consider an isotropic photon (boson) field with an arbitrary frequency spectrum trapped in an enclosure having perfectly reflecting walls and 
linear dimensions large compared with the wavelengths considered. Suppose now that we have for every frequencies $\omega, N_{T}$ atoms which have two energetic states separated by energy $\Delta E=\hbar \omega$. Let $N_{\mathrm{g}}$ and $N_{\mathrm{e}}$ are numbers of atoms in the ground and excited states, respectively. From statistical mechanics it is known that in the thermal equilibrium at the temperature $T$

$$
\frac{N_{\mathrm{e}}}{N_{\mathrm{g}}}=\exp (-\beta \Delta E)=\exp (-\beta \hbar \omega),
$$

where $\beta=1 / k T$. An atom in the ground state can absorb a photon and pass to the excited state and arialogously a photon can be emitted and the atom can pass from excited to the ground state.

Let $\vec{n}(q)$ is an averaged number of photons with a given frequency $\omega$ (statistical distribution function). Then the probability of absorption is

$$
P_{\mathrm{a}}=N_{\mathrm{g}}[\bar{n}(q)]_{q}|c|^{2}
$$

where $c$ is a constant.

The probability of emission is

$$
P_{\mathrm{e}}=N_{\mathrm{e}}[\bar{n}(q)+1]_{q}|c|^{2} .
$$

In the equilibrium $P_{\mathrm{a}}=P_{\mathrm{e}}$,

$$
N_{\mathrm{g}}[\bar{n}(q)]_{q}=N_{\mathrm{e}}[\bar{n}(q)+1]_{q} .
$$

Therefore

$$
\frac{[\bar{n}(q)]_{q}}{[\bar{n}(q)+1]_{q}}=\exp (-\beta \hbar \omega) .
$$

From this equation we get the distribution function

$$
\bar{n}(q)=\frac{1}{2 \ln q} \ln \left|\frac{1-\exp (-\beta \hbar \omega-\ln q)}{1-\exp (-\beta \hbar \omega+\ln q)}\right| .
$$

The spectral energy density is then

or

$$
U(\omega, q)=\bar{n}(q) \hbar \omega \frac{\omega^{2}}{\pi^{2} c^{3}}
$$

$$
U(x, q)=\frac{1}{2 \ln q} \ln \left|\frac{1-\exp (-x-\ln q)}{1-\exp (-x+\ln q)}\right| .
$$

This function has a singularity for

$$
x_{\mathrm{c}}=\ln q \text {. }
$$

For the limit $q=1$ in Eqs. (14) and (25) the non-deformed spectral energy density is recovered

$$
\bar{U}(x)=\frac{x^{3}}{\exp (x)-1} .
$$

This function is singularity-free.

Probably the reason for singularity of the $q$-deformed spectrum energy densities (14) and (25) is the fact that $q$-harmonic oscillator is equivalent to the nonlinear oscillator with a potential of the type

$$
V(x)=\sum c_{2 n} x^{2 n}
$$


where coefficients $c_{2 n}$ depend on $q$ and can be calculated numerically using, for example, Wentzel-Kramers-Brillouin method [9].

In order to understand the physical meaning of singularities we plan to study the $q$-deformation of a $\phi^{2}$ scalar field theory, which can be considered as an infinite sum of a harmonic oscillator [10]. The $q$-analogue of the $\phi^{2}$ theory is probably the nonlinear field theory of the type $\lambda \phi^{4}$, the critical behaviour of which has been extensively studied $[11,12]$.

\section{References}

[1] V.G. Drinfeld, Quantum Groups, Proc. Internat. Congr. of Mathematics, Berkeley, California, Vol. 1, Acad. Press, New York 1986, p. 798.

[2] M. Jimbo, Lett. Math. Phys. 10, 63 (1985).

[3] L.C. Biedenharn, J. Phys. A, Math. Gen. 22, L873 (1989).

[4] A.J. MacFarlane, J. Phys. A, Math. Gen. 22, 4581 (1989).

[5] V. Bužek, J. Mod. Optics 38, 801 (1991).

[6] Y.J. Ng, J. Phys. A, Math. Gen. 23, 1023 (1990).

[7] M.L. Ge, G. Su, J. Phys. A, Math. Gen. 24, L721 (1991).

[8] M.A. Martin-Delgado, J. Phys. A, Math. Gen. 24, L1285 (1991).

[9] D. Bonatsos, C. Daskaloyannis, K. Kokkotas, J. Phys. A, Math. Gen. 24, L795 (1991).

[10] L.H. Ryder, Quantum Field Theory, Cambridge University, Cambridge 1985.

[11] D. Amit, Field Theory, The Renormalization Group and Critical Phenomena, McGraw-Hill, New York 1978.

[12] R. Rajaraman, Solitons and Instantons, North-Holland, Amsterdam 1982. 